\title{
La Inteligencia Emocional y el Modelo Psicobiológico de la Personalidad de Eysenck
}

\author{
Emotional Intelligence and Eysenck's Psychobiological Model of Personality
}

\author{
Aldo Raúl Tesoro * \\ Lucas G. Gago Galvagno** \\ Carolina De Grandis***
}

\section{Resumen}

La presente investigación surge como resultado de la indagación sobre la Inteligencia Emocional (IE) a la luz de las diferentes dimensiones de la Personalidad propuestas por Eysenck. Para ello, se ha correlacionado la variable IE, medida a través del TMMS-24 (Fernández-Berrocal et al., 2004), con las diferentes dimensiones del Modelo Psicobiologico de Eysenck, a saber: Extraversión, Neuroticismo y Psicoticismo, obtenidas por medio del instrumento EPQRA (Squillace et al., 2013); ambos datos se extrajeron de una muestra compuesta por adultos de ambos sexos del Gran Buenos Aires y CABA., a quienes se les administró a su vez, una encuesta sociodemográfica, todo ello de manera virtual $(n=215)$. En cuanto a los resultados, se han obtenido correlaciones significativas positivas entre Atención Emocional y Neuroticismo, al igual que con Reparación Emocional y Extraversión. Asimismo, se han encontrado correlaciones significativas negativas entre Claridad Emocional con Neuroticismo y Psicoticismo, y en Reparación Emocional y Neuroticismo. Palabras clave: Inteligencia Emocional, Extraversión, Neuroticismo, Psicoticismo, Adultos.
Abstract
The present investigation arises as a result of the inquiry on Emotional Intelligence in the light of the different constructs of the Personality proposed by Eysenck. For this, the E.I. variable measured through TMMS- 24 (Fernández-Berrocal et al., 2004) has been correlated with the different variables in which the dimensions of the Personality are manifested, namely: Extraversion, Neuroticism and Psychoticism, obtained by means of the EPQ-RA instrument (Squillace

\footnotetext{
*Universidad Abierta Interamericana. Mail de contacto: aldo_tesoro@hotmail.com

**Consejo Nacional de Investigaciones Científicas y Técnicas ; Universidad Abierta Interamericana

***Consejo Nacional de Investigaciones Científicas y Técnicas. Centro Interdisciplinario de Investigaciones en Psicología Matemática y Experimental ; Universidad Abierta Interamericana DOI: https://doi.org/10.46553/RPSI.17.33.2021.p104-118
}

Fecha de Recepción: 23 de noviembre de 2020 Fecha de Aceptación: 27 de mayo de 2021 
et al., 2013); both data were extracted from a sample of adults of both sexes of Gran Buenos Aires and C.A.B.A., who were in turn administered a sociodemographic survey, all of them virtually $(n=215)$. Regarding the results, significant positive correlations have been obtained between Emotional Attention and Neuroticism, as well as with Emotional Reparation and Extraversion. Likewise, significant negative correlations were found between Emotional Clarity with Neuroticism and Psychoticism, and in Emotional Reparation and Neuroticism.

Keywords: Emotional Intelligence, Extraversion, Neuroticism, Psychoticism, Adults.

\section{Introducción}

Actualmente, existen dos marcos teóricos que explican la Inteligencia Emocional (IE): Los Modelos Mixto, que comprenden la IE como una mezcla de atributos vinculados con la personalidad, caracterizada por las habilidades sociales, la motivación y la capacidad cognitiva, necesarios para el éxito profesional (Pérez-Pérez y Castejón, 2006; Goleman, 1996); y los Modelos de Habilidad, que conciben la IE como una inteligencia propia del individuo independiente de los rasgos de personalidad, cuya función es percibir, asimilar, comprender y regular la información emocional a nivel cognitivo (Fernández-Berrocal y Pacheco, 2005; Mayer et al., 2000, citado en GarcíaFernández y Giménez-Mas, 2010).

Fernández-Berrocal et al. (2004) establecieron tres dimensiones de la IE: (a) Atención Emocional que es la tendencia de una persona a focalizar y reflexionar sobre sus emociones para maximizar su experiencia emocional (Salguero et al., 2009; Aritzeta y Gartzia, 2009). Los individuos atentos emocionalmente se caracterizan por estar alerta en todo momento en un intento por comprender sus sentimientos, lo cual es contraproducente si no está acompañado por una alta Claridad Emocional (Thayer et al., 2003, citado en Castillo et al., 2009); (b) Claridad Emocional, que es la percepción subjetiva sobre las propias emociones que nos permiten distinguirlas, comprendiendo el motivo, fundamento y consecuencia de un estado afectivo (Extremera y FernándezBerrocal, 2005; Salguero et al., 2009; Aritzeta y Gartzia, 2009); y (c) Reparación Emocional, que hace referencia a la creencia de poder interrumpir y regular los estados emocionales negativos, reemplazarlos por positivos y prolongarlos en el tiempo (Extremera y Fernández-Berrocal, 2005; Salguero et al., 2009; Aritzeta y Gartzia, 2009). Aquellas personas con mayor capacidad para diferenciar emociones tendrán mayores aptitudes para regularlas (Feldman-Barret et al., 2001), por medio de estrategias centradas en la disminución o exacerbación de estados emocionales (Gross \& John, 2003).

Eysenck (1985, citado en Ortet i Fabregat et al., 2008) definió la personalidad como una organización más o menos estable y duradera de la conducta conativa, afectiva, cognitiva y física de una persona que determina su adaptación al ambiente, y la cual es el resultado de una integración jerárquica compuesta por dimensiones bases que determinan ciertos rasgos, que producen a su vez respuestas habituales y específicas. En tanto, propuso un modelo tridimensional en el que los individuos pueden ser descritos: (a) Extraversión, que evalúa la necesidad 
de un individuo a estimularse, y en su base biológica se encuentra el sistema reticular ascendente, cuya función es activar o no la corteza cerebral (Eysenck \& Eysenck, 1994, citado en Ortei i Fabregat et al., 2008; Bermúdez-Moreno et al., 2017), lo que explicaría la preferencia de los extrovertidos a buscar experiencias que aumenten la activación cortical, y la propensión de los introvertidos, que alcanzan niveles óptimos con menor estimulación, a la rutina (Schmidt et al., 2010). De acuerdo a Eysenck (1987, citado en Squillace et al. 2013), los rasgos de esta dimensión son: Sociabilidad, vivacidad, actividad, asertividad, búsqueda de sensaciones, inquietud, despreocupación, dominación, espontaneidad, y aventura; (b) Neuroticismo, que mide el grado de estrés y las respuestas emocionales del sujeto frente a las circunstancias que alteran su equilibrio interno y la predisposición a padecer trastornos de ansiedad o trastornos del ánimo (Schmidt et al., 2010). Las personas cuya puntuación es elevada, tenderán a reaccionar de manera excesiva frente a los estímulos, presentando oscilaciones de humor, demoras en la recuperación, ansiedad, preocupación, depresión, pesimismo, sentimiento de culpa, aflicción, y baja autoestima (Bermúdez Moreno et al., 2017; Eysenck \& Eysenck 1994, citado en Ortei i Fabregat et al., 2008; Schmidt, 2010; Squillace et al. 2013); y 3) Psicoticismo, que estima la dureza emocional (Schmidt et al., 2010). Un individuo con alto Psicoticismo es proclive a la agresividad, frialdad, impulsividad, egocentrismo, $\mathrm{y}$ poca empatía, mientras que, a puntuaciones bajas, serán patentes conductas altruistas, empáticas y cooperativas (Eysenck, 1990, citado en Schmidt et al., 2010; Squillace et al. 2013; Bermúdez Moreno et al., 2017).
A la luz de la teoría, sería interesante indagar las relaciones que pueden llegar a establecerse entre el Modelo Psicobiologico de la Personalidad y la IE, examinando que papel ocupa estaúltima. ¿Es más bien un rasgo o una habilidad? Sabemos gracias a estudios a partir de los modelos Big Five y EQ-i, que la dimensión Neuroticismo correlaciona negativamente con las dimensiones de la Inteligencia Emocional, siendo la variable que mejor la predice (Fortes-Vilaltella et al., 2013). Mesa Jacobo (2015), Parodi-Úbeda (2015), y Parodi-Úbeda et al. (2017), han arribado a la misma conclusión. Asimismo, se han hallado correlaciones positivas entre Extraversión e Inteligencia Emocional (Bernarás et al., 2011; Fortes-Vilaltella et al., 2013). En estudios que hayan utilizado el TMMS-24, se han observado correlaciones negativas entre Neuroticismo con Claridad Emocional y Reparación Emocional, así como correlaciones positivas entre Extraversión con Claridad y Regulación Emocional (Perandones González y Castejón Costa, 2007; Pérez-Pérez, 2013).

En cuanto a la Inteligencia Emocional según Edad y Género, Bernarás et al. (2011) concluyen que no existen diferencias. No obstante, Mestre et al. (2004), Aragón et al. (2008), y Núñez et al. (2008) observaron que las mujeres son más hábiles al momento de manipular los estados afectivos. Colorado y Bisval (2015), evidenciaron que estas diferencias se daban en Atención Emocional en los hombres, y en Claridad Emocional en las mujeres. A su vez, Ugarriza y Pajares-Del-Águila (2001) y Navarro et al. (2009), observaron que existen una diferencia significativa entre adultos (edad media: 65.7) y jóvenes (edad media: 22.14) a favor de los primeros, y que la IE 
se incrementa con la edad, descendiendo a partir de los cincuenta años. En lo que respecta a la Personalidad, Bernarás et al. (2011) tampoco encontró diferencias significativas en función del sexo y la edad. Estos resultados se contradicen con los encontrados por Garaigordobil et al. (2009), Eysenck \& Porrata (1984) y Giaquinto (2019), donde se observó una prevalencia de Neuroticismo en el género femenino; estos a su vez se contradicen con los hallazgos de Meza Jacobo (2015), quien observó que los hombres obtienen índices más altos en Inestabilidad Emocional, mientras que las mujeres presentan medidas más altas en Extraversión.

En resumen, se busca analizar las relaciones significativas entre las dimensiones de la Inteligencia Emocional y las dimensiones de la Personalidad según el modelo Psicobiológico de Eysenck., a la vez que se analizan las diferencias significativas de estas dimensiones en función de la Edad y el Género, con la finalidad de ampliar el conocimiento que se posee actualmente sobre el papel de la IE como $<<$ rasgo $>>$ o $<<$ habilidad $>>$, facilitando el desarrollo de intervenciones a nivel individual y educacional, en paralelo a resaltar su importancia para la comunidad y sus instituciones. Basándonos en los estudios previos, se espera encontrar: (a) correlación significativa y positiva entre Extraversión con Claridad y Reparación Emocional; (b) correlación significativa y positiva entre Neuroticismo y Atención Emocional, y correlación significativa y negativa entre Neuroticismo con Claridad y Reparación Emocional; (c) correlación significativa y negativa entre Psicoticismo y Claridad Emocional; (d) diferencia significativa en las dimensiones de la Inteligencia Emocional y la Personalidad en función del Género; (e) diferencia no significativa en las dimensiones de la Inteligencia Emocional y la Personalidad en función de la Edad.

\section{Método}

\section{Participantes}

El tipo de muestreo respondió a un carácter no probabilístico, intencional, y por bola de nieve. Estuvo formado por 215 personas entre los 18 y 65 años, con una Media de 29,59 y un Desvió Típico de 10,42. Del total, 64 pertenecieron al género masculino en un ratio de edad entre los 18 y 58 años, de los cuales 54 residían en Buenos Aires y los 10 restante en Ciudad de Buenos Aires (CABA); los otros 151 individuos, de género femenino en un espectro de edad entre los 18 y 65 años, 130 pertenecieron a Buenos Aires, y 21 a CABA. El total de la muestra correspondió a una administración virtual.

\section{Instrumentos}

\section{Cuestionario Sociodemográfico}

Se indagó sobre la edad, el género, y el lugar de residencia.

\section{Escala de Inteligencia Emocional (TMMS-} 24, Salovey y Mayer, 1995, Validación y Adaptación Española por: FernándezBerrocal et al., 2004)

Instrumento psicométrico autoadministrable que evalúa el meta conocimiento de los estados emocionales mediante 24 ítems, en concreto, las destrezas con las que podemos ser conscientes de nuestras propias emociones, así como de nuestra capacidad para regularlas. La TMMS-24 contiene tres dimensiones claves 
de la IE con 8 ítems en cada una de ellas: Atención emocional (1-8), Claridad de sentimientos (9-16) y Reparación emocional (17-24), cuyas opciones de respuesta es una escala Likert de cinco puntos donde: $1=$ Totalmente en Desacuerdo, y $5=$ Totalmente de Acuerdo. El coeficiente Alfa de Cronbach es de .90 para Atención y Claridad, y .86 para Reparación. Para las dimensiones del TMMS-24 de la presente muestra $(\mathrm{n}=215)$, se obtuvo una fiabilidad de: $\alpha=.82$ para Atención; $\alpha=.89$ para Claridad; $\alpha=.85$ para Reparación.

\section{Cuestionario de Personalidad (EPQ-RA,} Eysenck \& Eysenck, 2001, Validación Argentina por: Squillace et al., 2013)

Instrumento

psicométrico

autoadministrable que evalúa las 3 dimensiones de la personalidad propuestas porH.J.Eysenck: Neuroticismo, Extraversión y Psicoticismo. Este inventario consta de 42 ítems los cuales se responden de forma dicotómica, Sí o No. El coeficiente Alpha de Cronbach es de .84 para Neuroticismo, .77 para Extraversión, y .66 para Psicoticismo. La fiabilidad de EPQ-RA de la presente muestra $(\mathrm{n}=215)$, arrojó: $\alpha=.8$ para Neuroticismo; $\alpha=.77$ para Extraversión; $\alpha$ $=.58$ para Psicoticismo.

\section{Procedimiento}

A través de un consentimiento informado, se administró de manera voluntaria en Buenos Aires y CABA, los cuestionarios a completar: Sociodemográfico, EPQ-RA y TMMS-24, todos ellos realizados de manera virtual a través de Google Forms. Para ello, se procedió a emular los cuestionarios físicos en la plataforma virtual; a su vez, se solicitó a los participantes su contribución a través de las redes sociales (Facebook y WhatsApp) para que lo distribuyan entre los allegados que cumplan con los criterios de inclusión pertinentes. Se explicó, además, en qué consistía la investigación a la vez que se le garantizó el anonimato. Previo a la salida a campo, se realizó una prueba piloto a 5 personas de $15,25,35,45$, y 55 años respectivamente, con la finalidad de observar las posibles dudas, consultas, inquietudes, o inconvenientes que podrían llegar a presentarse, y así solucionarlo.

Los datos e información obtenidos fueron volcados y procesados por el software Statistical Package for the Social Sciences (SPSS) en su versión 22 de IBM.

La variable Edad fue recodificada de acuerdo a los criterios de Nassar y Abarca (1983, citado en Alpízar Díaz, 2005), quedando constituida por tres rangos: Adulto joven (entre los 18 y 25 años), Adulto medio (entre 26 y 45 años), y Adulto tardío (entre 46 y 65 años).

\section{Resultados}

\section{Análisis de datos}

Se realizó una prueba de normalidad para las dimensiones de la Inteligencia Emocional y de la Personalidad a través de la prueba de Kolmogorov-Smirnov: En la misma se observó que Claridad y Reparación Emocional se distribuían de manera anormal y Atención Emocional de manera normal. Asimismo, Extraversión, Neuroticismo, y Psicoticismo se distribuyeron de manera anormal. De acuerdo a esto, se utilizaron las pruebas no paramétricas de Rho de Spearman para las correlaciones, y H de Kruska Wallis para las comparaciones de grupo. 


\section{Caracterización de la muestra}

La variable Edad, recodificada de acuerdo a los criterios de Nassar y Abarca, obtuvo una distribución de 112 sujetos en la Adultez Joven (52,1\% de la muestra), 84 sujetos en la Adultez Media (39,1\% de la muestra), y 19 sujetos en la Adultez Tardía (8,8\% de la muestra). La distribución de la variable Género, quedo conformada por 64 sujetos masculinos (29,8\% de la muestra) y 151 sujetos femeninos (70,2\% de la muestra). Por último, la distribución muestral de la variable
Residencia, se organizó en 184 sujetos para Gran Buenos (85,6\% de la muestra), y 31 para CABA $(14,4 \%$ de la muestra).

\section{Análisis de correlación entre variables}

Tal como se observa, Atención Emocional correlacionó de manera positiva y débil con Neuroticismo (Rho = .35). A su vez, Claridad Emocional correlacionó de manera negativa y débil tanto con Neuroticismo (Rho = -.33), como con Psicoticismo (Rho $=-.16)$. Finalmente, Reparación Emocional correlacionó de manera positiva y débil

Tabla 1

Descripción de la muestra según Variables Sociodemográficas.

\begin{tabular}{lcc}
\hline Variable Sociodemográfica & Frecuencia & Porcentaje \\
\hline Edad según Nassar y Abarca & & \\
Adulto joven & 112 & $52.10 \%$ \\
Adulto medio & 84 & $39.10 \%$ \\
Adulto tardío & 19 & $8.80 \%$ \\
\hline Genero & & \\
Masculino & 64 & $29.80 \%$ \\
Femenino & 151 & $70.20 \%$ \\
\hline Residencia & & \\
Gran Buenos Aires & 184 & $86.60 \%$ \\
CABA & 31 & $14.40 \%$ \\
\hline
\end{tabular}

\section{Tabla 2}

Correlación entre dimensiones de Inteligencia Emocional y dimensiones de la Personalidad

\begin{tabular}{lccc}
\hline Inteligencia Emocional & Extraversión & Neuroticismo & Psicoticismo \\
\hline Atención Emocional & .03 & $.35^{* *}$ & -.08 \\
Claridad Emocional & $.19^{* *}$ & $-.33^{* *}$ & $-.16^{*}$ \\
Reparación Emocional & $.30 * *$ & $-.45^{* *}$ & -.07 \\
\hline
\end{tabular}

Nota: Prueba utilizada: Rho de Spearman 
con Extraversión $(\mathrm{Rho}=.30)$, y de manera negativa y moderada con Neuroticismo (Rho $=-.45$ ).

\section{Análisis de comparación de grupos} Inteligencia Emocional y Personalidad en función de la Edad

No se obtuvieron diferencias significativas en función de la variable Edad
Inteligencia Emocional y Personalidad en Función del Género

Se obtuvieron diferencias significativas en la dimensión de la personalidad Extraversión $(\mathrm{p}<.03)$ en función de la variable Genero. Las mujeres presentaron mayor extraversión que los hombres.

\section{Tabla 3}

Diferencias entre Edad en Inteligencia Emocional y Personalidad

\begin{tabular}{lccc}
\hline Inteligencia Emocional y Personalidad & Edad & $\begin{array}{c}\text { Rango } \\
\text { Promedio }\end{array}$ & $\begin{array}{c}\text { Sig. } \\
\text { Asintót }\end{array}$ \\
\hline Atención Emocional & Adulto joven & 115.18 & \\
& Adulto medio & 100.20 & .21 \\
& Adulto tardío & 100.16 & \\
\hline Claridad Emocional & Adulto joven & 99.37 & \\
& Adulto medio & 114.95 & .07 \\
& Adulto tardío & 128.16 & \\
\hline \multirow{2}{*}{ Reparación Emocional } & Adulto joven & 100.79 & \\
& Adulto medio & 112.64 & .11 \\
& Adulto tardío & 130.00 & \\
\hline \multirow{2}{*}{ Extroversión } & Adulto joven & 100.96 & \\
& Adulto medio & 113.92 & .18 \\
& Adulto tardío & 123.37 & \\
\hline Neuroticismo & Adulto joven & 115.67 & \\
& Adulto medio & 101.43 & .13 \\
& Adulto tardío & 91.82 & \\
\hline Psicoticismo & Adulto joven & 111.05 & \\
& Adulto medio & 110.54 & .09 \\
& Adulto tardío & 78.79 & \\
\hline
\end{tabular}

Nota: Prueba utilizada: H de Kruska

Wallis 


\section{Conclusiones}

En las últimas décadas, el concepto de Inteligencia Emocional ha cobrado un interés creciente dentro de los círculos académicos. El modelo de Goleman y el de Mayer-Salovey, dominan el estudio de las emociones, y la gran divergencia que los separa, radica en otra variable psicológica, la personalidad, siendo la IE contemplada para unos como un rasgo, y para otros, como una habilidad independiente. Esta contrariedad, ha sido motivo y fundamento de la presente investigación: Analizar si existen relaciones significativas entre las dimensiones de la Inteligencia Emocional y las dimensiones de la Personalidad. Tal como puede observarse en las hipótesis propuestas, es posible desprender para cada dimensión de la Personalidad, un perfil esperable de Inteligencia Emocional. Así es que, para los sujetos Extravertidos, era presumible a priori, una correlación significativa y positiva con Claridad Emocional y Reparación Emocional. Aunque la primera correlación se refuto $(\mathrm{p}<.56)$, la segunda, no obstante, si pudo comprobarse $(\mathrm{p}<.00)$, aunque de manera débil $(\mathrm{r}=.30)$, tal como se visualiza en la Tabla 2. Estos resultados coinciden con los obtenidos por AguilarLuzon y Landa (2009), Bernarás et al. (2011), Fortes-Vilaltella et al. (2013), PérezPérez (2013) y Mesa Jacobo (2015). Con estos resultados, es plausible apuntar a la idea de que, una buena salud psicoemocional está respaldada a su vez por la personalidad base. No son pocos los estudios que comprueban la relación positiva entre Bienestar Psicoemocional y Extraversión, así como Malestar Psicoemocional y Neuroticismo. Villanueva Acosta (2016), comprobó que el Neuroticismo es una dimensión que predice desequilibrios a nivel afectivo, y Fortes-Vilaltella et al. (2013) observó que es la dimensión con mayor poder predictivo negativo de la IE, y en menor medida, la Extraversión, como factor predictivo positivo. Otros autores, a su vez, han confirmado la relación positiva existente entre la IE, y en específico la Reparación Emocional, con la Felicidad, el Bienestar Subjetivo, las Relaciones Intra e Interpersonales, la Satisfacción Vital, la Resiliencia y la Salud Mental en general (Fernández-Berrocal y Extremera, 2009; Fernández-Berrocal, 2019; Veloso-Besio et al., 2013; Ferragut y Fierro, 2012). Estos resultados, sustentan la idea de que una elevada Extraversión, es decir, la tendencia a ser sociable, despreocupado, vivaz, abierto, asertivo, espontáneo, entre otros, supone en los individuos una garantía de salud mental, al contar con mayores capacidades en el manejo de estrategias afrontamiento en la regulación positiva del estrés, orientadas a la creatividad, sublimación y crecimiento personal; es decir, mayor Reparación Emocional.

Para el sujeto Neurótico, el perfil de Inteligencia Emocional creado previo a los resultados, indicaba correlación significativa y positiva con Atención Emocional y correlaciones significativas y negativas con Claridad y Regulación Emocional. Las tres hipótesis han sido confirmadas, tal como se presenta en la Tabla 2, al obtener una significancia menor a .005 , aunque de manera débil (a excepción de Reparación Emocional cuya correlación obtuvo una fuerza moderada, Rho $=-.45$ ). Estos resultados coinciden con los hallados por Perandones González y Castejón (2007), Aguilar-Luzon y 
Landa (2009), Bernarás et al. (2011), FortesVilaltella et al. (2013), Pérez-Pérez (2013), Mesa Jacobo (2015), Parodi-Úbeda (2015) y Parodi-Úbeda et al. (2017). Estos autores no solo corroboran las relaciones negativas entre Neuroticismo y la IE, sino, además, el valor predictivo de esta dimensión de la personalidad sobre la salud psicoemocional del sujeto, al que lo predispone a una concatenación de patologías asociadas a la ansiedad, la depresión, el consumo de drogas, el suicidio, los pensamientos rumiativos, $\mathrm{y}$ estrategias de afrontamiento poco o nada eficientes (Fernández-Berrocal et al., 2011, citado en Díaz, 2013; Fernández-Berrocal, 2019). Estas problemáticas son el producto de las dimensiones de la IE trabajando en conjunto para generar en todos los ámbitos emocionales del individuo una Inestabilidad Afectiva: Los bajos índices de Claridad Emocional que producen una dificultad en el discernimiento emocional que nos atraviesa, conducen a la imposibilidad de repararlas (bajos índices de Reparación Emocional), lo que conlleva a sesgos atencionales excesivos (Alta Atención Emocional) con la finalidad de poder mantener el control minino- sobre lo que nos afecta (Hertel et al., 2009, citado en Lizeretti y Extremera, 2011; Hervás y Vázquez, 2006; Lizeretti y Núria García, 2014). En base a ello, se sostiene que los individuos con Inestabilidad Emocional, cuya manifestación típica se corresponde a una sintomatología ansiogena, culposa y con oscilaciones en el estado de ánimo, son propensos a un estado de alerta hiperactivo sobre las emociones que lo circundan, focalizando sobre ellos y maximizándolos; esta relación a su vez, adquiere un matiz aún más negativo, si partimos de la idea de que su exagerada
Atención Emocional va acompañada de una pobre Claridad Emocional, es decir, una incapacidad en el reconocimiento de la emoción y su desencadenante, debido tanto a los rasgos de personalidad, como la ansiedad, la preocupación, la alteración de la homeostasis afectiva, y a la hipervigilancia emocional a la que se somete, cuyo mayor caudal de emociones presente, dificulta en mayor medida, la claridad de las mismas. La combinación de una alta Atención Emocional y baja Claridad Emocional desencadena una respuesta del organismo frente a los estados afectivos, limitada, representada por menores recursos al momento de la Reparación Emocional, siendo por debajo de la media, competentes al momento de interrumpir emociones negativas $o$ potenciando las positivas, y reaccionando de manera excesiva a los estímulos, con mayor estrés, y una tardanza mayor en la posterior recuperación.

Por último, se postuló, que la dimensión Psicoticismo correlacionaría significativa y negativamente con Claridad Emocional. En la Tabla 2, puede observarse que, en efecto, H3 se confirma $(\mathrm{p}<.01)$, aunque con una fuerza de correlación débil $($ Rho $=-.16)$. No existen estudios previos que vinculen la dimensión $\mathrm{P}$ de Eysenck con las dimensiones de la IE medida a través del TMMS-24 u otro instrumento, lo que dificulta elaborar una posible explicación. No obstante, y gracias a la bibliografía teórica que circunda tanto al constructo Psicoticismo como al de Claridad Emocional, podemos pensar esta relación negativa como una característica de esta dimensión; mientras que lo habitual para los Extravertidos es mantener una Reparación Emocional alta que fecunda un bienestar 
a nivel psicológico, y lo regular para los Neuróticos es una constitución singular de la IE caracterizada en general por una deficiencia en todas las dimensiones (Alta AE, y bajas CE Y RE) lo que ocasiona una pobreza en la percepción, la comprensión y la elaboración de las situaciones estresantes y las emociones, en los sujetos con alta Dureza Emocional, es esperable, por los elementos que la constituyen (impulsividad, frialdad, poca empatía, agresividad, impersonalidad e indiferencia) una carencia en las facultades empáticas, o sea, una incompetencia en la Claridad Emocional, cuyo valor reside necesariamente, en la destreza para distinguir, conocer, e interpretar tanto las propias emociones como las de los demás, dando como resultado, una tendencia en estos individuos a encerrarse en sí mismos, adoptando una posición egocéntrica, con poca conciencia de los demás, y una incapacidad para la expresión de afecto.

De acuerdo a la Hipótesis 4, en la que se establece que existen diferencias en las dimensiones de la Inteligencia Emocional en función del Género, se refuta, al observarse en la Tabla 3 que no hay significación estadística. Estos resultados coinciden con los encontrados por Bernarás et al. (2011), pero no concuerdan con los obtenidos por Mestre et al. (2004), Fortes-Vilaltell et al. (2013), Aragón et al. (2008) y Colorado y Bisval (2015). Una posible explicación podría hallarse en los instrumentos utilizados para medir la IE en las investigaciones llevadas a cabo por Mestre et al., y FortesVilaltella et al., que, a diferencia de los auto informes, miden las competencias emocionales a través de una serie de pruebas de rendimiento. Por su parte, Rojas (2014), nos dice que el Autocontrol correlaciona de manera significativa y positiva con el nivel de instrucción de los padres, y siguiendo esta línea, Núñez et al. (2008) explican que las diferencias en las dimensiones de la IE en función del Genero pueden deberse tanto a la instrucción de los padres en el manejo afectivo, como a los estereotipos sociales que pesan sobre el sexo femenino, y al que tienden a mirarlo como más empático $\mathrm{y}$ emocional. Hoy en día, y en pleno 2019/20 vivimos en una revolución cultural que ha dejado atrás muchos de los mitos que rodeaban a las mujeres y a los hombres; por su parte, el presente estudio indica que, en efecto, tanto hombre como mujeres son igualmente capaces de percibir, entender y manejar sus estados afectivos.

En la Hipótesis 4, también se conjetura que existirán diferencias en las dimensiones de la Personalidad en función del Género; como se observa en la Tabla 4, los sujetos Femeninos puntúan más alto en Extraversión $(\mathrm{p}<.03)$ en comparación a los Masculinos, por lo que la H4 queda confirmada. Estos resultados coinciden con los encontrados por Eysenck y Porrata (1984), McCrae et al. (2002), y Meza Jacobo (2015), pero no concuerdan con los encontrados por Garaigordobil et al. (2009) y Giaquinto (2019), quienes encontraron una predominancia de Neuroticismo en la muestra femenina, y con Bernarás et al. (2011), quienes no encontraron diferencia significativa alguna. En otras palabras, lo que a la presente investigación refiere, entendemos que la muestra Femenina entre 18 y 65 años de edad de Bs As y CABA., presenta características más desarrolladas, o al menos así lo perciben, en torno a sociabilidad, vivacidad, actividad, asertividad, búsqueda de sensaciones, despreocupación, 
dominación, espontaneidad, y aventura, en comparación a la muestra Masculina entre 18 y 65 años de edad de Bs As y CABA.

La Hipótesis 5, donde se establece que no existen diferencias significativas en las dimensiones de la Inteligencia Emocional en función de la Edad, se observa en la Tabla 3 que, en efecto, la IE es independiente a esta variable. Estos resultados coinciden con los encontrados por Bernarás et al. (2011), y se contradicen con los estudios hechos por Ugarriza y Pajares-Del-Águila (2001) y Navarro et al. (2009) en los que concluyeron que a medida que se envejece se desarrolla la inteligencia emocional. Las conclusiones opuestas a las que se ha arribado en diversos estudios, incluido el propio, puede deberse al hecho de que la IE como habilidad, no depende tanto de la edad en sí, sino más bien de otros factores, como la personalidad base, el mindset (mentalidad), o el desarrollo a través del aprendizaje que se obtiene por medio de diferentes técnicas o prácticas. En lo que respecta a las dimensiones de la Personalidad y su falta de diferencias significativas en función de la Edad, se confirma en efecto, que la Personalidad es independiente a esta variable (Ver Tabla $3)$. Estos resultados coinciden con los encontrados por Bernarás et al. (2011) y Mesa Jacobo (2015), lo que refuerza la idea de que, una dimensión de la Personalidad ya instaurada (Extraversión, Neuroticismo, Psicoticismo), no es susceptible de grandes cambios a lo largo del tiempo.

En resumen, se evidencia que la Inteligencia Emocional suele encontrar un correlato directo con los rasgos de Personalidad, o sea que, los rasgos de Personalidad podrían predecir la futura Inteligencia Emocional de un individuo.
No hay seguridad en qué medida se de esta direccionalidad en la dimensión de Psicoticismo, pero es posible sostener con mayor fuerza, que los sujetos Extravertidos tienden a poseer una mayor Reparación Emocional y que los Neuróticos son proclives a dar un Perfil caracterizado por alta Atención Emocional, y menor Claridad y Reparación Emocional.

En cuanto a las limitaciones de la presente investigación, es necesario considerar la desproporción de participantes en función del género, y el número total de la muestra, que, al ser reducido, no es representativo de la población. Otra limitación, fue el escaso estado del arte que relacione Inteligencia Emocional medida a través del TMMS-24 y las dimensiones de la Personalidad evaluadas mediante el EPQRA, en población adulta, lo que dificulto el recuento de investigaciones actuales hechas en Argentina o países de habla hispana. Asimismo, la imposibilidad de verificar la influencia de sesgos cognitivos, como recordar o no sucesos, exagerar o disminuir el efecto de un evento, o la deseabilidad social en las respuestas de los encuestados, y la falta de una escala de validez en el TMMS24 , son factores a tener en cuenta.

Finalmente, y con el fin de continuar ampliando los resultados empíricos obtenidos, se podría recomendar el uso de diversas fuentes de información no psicométricas, como entrevistas que recopilen información sobre la biografía de los sujetos, sus vínculos, traumas, etc., y técnicas proyectivas que informen sobre los aspectos más inconscientes del individuo que no pueden captarse con cuestionarios. Además, es interesante el análisis de los estilos de apego infantil en un comienzo, con 
el objetivo de observar cómo incide sobre la gestión de las emociones en el infante. Es importante a su vez, realizar investigaciones de Inteligencia Emocional en Ancianos de las que no existe gran cantidad, y estudios que versen sobre las relaciones entre la IE y las diversas psicopatologías, como los T. de Estado de Ánimo, T. de Ansiedad, y T. Bipolares, y como puede incidir positivamente sobre la gestión de las emociones la meditación, el ejercicio físico, o la religión, entre otros.

\section{Referencias}

Aguilar-Luzon, M., y Landa, J. M. A. (2009). Relación entre inteligencia emocional percibida, personalidad y capacidad empática en estudiantes de enfermería. Behavioral Psychology/Psicología Conductual, 17(2), 351-365.

Alpízar Díaz, W. A. (2005). Hacia la construcción del concepto integral de adultez. Revista Enfermería actual de Costa Rica, (10), 1-4. doi: 10.15517/revenf.v0i10.10704

Aragón, R. S., Franco, B. E. R., y Chávez, E. C. (2008). Evaluación psicológica del entendimiento emocional: Diferencias y similitudes entre hombres y mujeres. Revista Iberoamericana de Diagnóstico y Evaluación Psicológica, 26(2), 193-216. 130

Aritzeta,A.A.,y Gartzia, L. G. (2009). Cultura Organizacional e Inteligencia Emocional. En Fernández-Berrocal (Ed.), Avances en el estudio de la inteligencia emocional (pp. 167172). Fundación Marcelino Botín

Bermúdez-Moreno, J., Pérez Garcia, A. M., y SanJuan Suarez, P. (2017). Psicología de la personalidad: Teoría e investigación. Volumen I. Madrid, España: UNED

Bernarás, E., Garaigordobil, M., y De las
Cuevas, C. (2011). Inteligencia emocional y rasgos de personalidad. Influencia de la edad y el género durante la edad adulta y la vejez. Boletín de Psicología, 103, 75-88.

Castillo, R., Fernández-Berrocal, P., Extremera, N., y Gonzales, V., (2009). La relación de la Atención Emocional y el Índice de Masa Corporal en directivos Españoles. En Fernández-Berrocal (Ed.), Avances en el estudio de la inteligencia emocional (pp. 217222). Fundación Marcelino Botín

Colorado, Y. S., y Bisval, C. W. (2015). Habilidades emocionales en una muestra de estudiantes universitarios: las diferencias de género. Educación y Humanismo, 17(28), 119-132. doi: 10.17081/ eduhum.17.28.1170

Díaz, J. J. (2013). Reseña teórica de la inteligencia emocional: modelos e instrumentos de medición. Revista cientifica, 1(17), 10-32. doi: $10.14483 / 23448350.4505$

Extremera, N., y Fernandez-Berrocal, P. (2005). Inteligencia emocional percibida y diferencias individuales en el meta-conocimiento de los estados emocionales: una revisión de los estudios con el TMMS. 
Ansiedad y estrés, 11(2), 101-122.

Eysenck, S. B. G. y Porrata J. L. (1984). Un Estudio Transcultural de Personalidad: Puerto Rico e Inglaterra. Revista Latinoamericana de Psicología, 16(3), 355-372.

Feldman-Barret, B. L., Gross, J. J., Conner, T. C. \& Benvenuto, M. (2001). Knowing what you're feeling and knowing what to do about it: Mapping the relation between emotion differentiation and emotion regulation. Cognition and Emotion, 15, 713-724. doi: 10.1080/02699930143000239

Fernández-Berrocal, P. (2019). Inteligencia Emocional. Aprender a gestionar las emociones. Buenos Aires, Argentina: Salvat

Fernández-Berrocal, P., Extremera, N., \& Ramos, N. (2004). Validity and reliability of the Spanish modified version of the Trait Meta-Mood Scale. Psychological reports, 94(3), 751-755. doi: 10.2466/ pr0.94.3.751-755

Fernández-Berrocal, P., y Extremera, N. (2009). La inteligencia emocional y el estudio de la felicidad. Revista interuniversitaria de formación del profesorado, (66), 85-108.

Fernández-Berrocal, P., y Pacheco, N. E. (2005). La Inteligencia Emocional y la educación de las emociones desde el Modelo de Mayer y Salovey. Revista Interuniversitaria de Formación del profesorado, 19(3), 63-93.

Ferragut, M., y Fierro, A. (2012). Inteligencia emocional, bienestar personal y rendimiento académico en preadolescentes. Revista latinoamericana de psicología, 44(3), 95-104.

Fortes-Vilaltella, M., Oriol, X., Filella, G., Del Arco, I., \& Soldevila, A. (2013). Inteligencia emocional y personalidad en las diferentes áreas de conocimiento de los estudiantes universitarios. Revista Electrónica Interuniversitaria de Formación del Profesorado, 16(1), 109-120. doi: 10.6018/reifop.16.1.179471

Garaigordobil, M., Aliri, J. y Fontaneda. I. (2009). Bienestar psicológico subjetivo: diferencias de sexo, relaciones con dimensiones de personalidad y variables predictoras. Behavioral Psychology / Psicología Conductual, 17(3), pp. 543-559.

García-Fernández, M., y Giménez-Mas, S. I. (2010). La inteligencia emocional y sus principales modelos: propuesta de un modelo integrador. Espiral. Cuadernos del profesorado, 3(6), 43-52. doi: 10.25115/ecp.v3i6.909

Giaquinto, L. (2019). El Modelo Psicobiologico de la Personalidad y los Estios de Apego (Tesis de pregrado). Universidad Abierta Interamericana, Buenos Aires, Argentina.

Goleman, D. (1996). Inteligencia Emocional. Barcelona: Kairós.

Gross, J. J., \& John, O. P. (2003). Individual differences in two emotion regulation processes: implications for affect, relationships, and wellbeing. Journal of personality and social psychology, 85(2), 348-362. doi: 10.1037/0022-3514.85.2.348 
Hervás, G. y Vázquez, C. (2006). La regulación afectiva: Modelos, investigación e implicaciones para la salud mental y física. Revista de Psicología General y Aplicada, 59, 9-32.

Lizeretti, N. P, y Núria García (2014). Crisis, depresión e Inteligencia Emocional. PsiAra - Col-legi Oficial de Psicologia de Catalunya.

Lizeretti, N., \& Extremera, N. (2011). Emotional intelligence and clinical symptoms in outpatients with generalized anxiety disorder (GAD). Psychiatric quarterly, 82(3), 253-260. doi: 10.1007/ s11126-011-9167-1

McCrae, R. R., Costa, P. T., Jr., Terracciano, A., Parker, W. D., Mills, C. J., De Fruyt, F., \& Mervielde, I. (2002). Personality trait development from age 12 to age 18: Longitudinal, cross-sectional and cross-cultural analyses. Journal of Personality and Social Psychology, 83(6), 1456-1468. doi: 10.1037/00223514.83.6.1456

Mesa Jacobo, J. R. (2015). Inteligencia emocional, rasgos de personalidad $e$ inteligencia psicométrica en adolescentes. (Tesis Doctoral) Universidad de Murcia, España.

Mestre, J. M., Bozal, R. G., y Lim, N. (2004). Inteligencia emocional: ¿A qué pueden deberse las diferencias respecto a la variable género? Motivos, emociones y procesos representacionales: de la teoría a la práctica. Departamento de Psicología Básica (pp. 313-324).

Navarro, B., Latorre, J. M. y Ros, L.
(2009). Inteligencia Emocional Autoinformada en la vejez, un estudio comparativo con el TMMS-24. En Fernández-Berrocal (Ed.), Avances en el estudio de la Inteligencia Emocional (pp. 331335). Fundación Marcelino Botín.

Núñez, M. T. S., Fernández-Berrocal, P., Rodríguez, J. M., y Postigo, J. M. L. (2008). ¿Es la inteligencia emocional una cuestión de género? Socialización de las competencias emocionales en hombres y mujeres y sus implicaciones. Electronic journal of research in educational Psychology, 6(2), 455-474. doi: 10.25115/ejrep.v6i15.1287

Ortet i Fabregat, G., Ibáñez Ribes, M., Moro Ipola, y Silva Moreno (2008). Manual del Cuestionario Revisado de Personalidad de Eysenck. Madrid, España: TEA Ediciones.

Parodi-Úbeda, A. I. (2015). Inteligencia emocional y personalidad: Factores predictores del rendimiento académico (Tesis Doctoral). Universidad de Murcia, España.

Parodi-Úbeda, A. P., Lillo, V. B., García, C. F., y Melero, M. J. R. (2017). La relación entre la Inteligencia Emocional y la Personalidad en estudiantes de Educación Secundaria. International Journal of Developmental and Educational Psychology, 2(1), 137-143. doi: 10.17060/ijodaep.2017.n1.v2.926

Perandones González, T. M., y Castejón, J. L. (2007). Estudio correlacional entre personalidad, inteligencia emocional y autoeficacia en profesorado de educación 
secundaria y bachillerato. $V$ Congreso Internacional "Educación y Sociedad", 1-8.

Pérez-Pérez, N. (2013). Aportación de la inteligencia emocional, personalidad e inteligencia general al rendimiento académico en estudiantes de enseñanza superior (Tesis Doctoral). Universidad de Alicante, España.

Pérez-Pérez, N. y Castejón, J.L. (2006). Relaciones entre la inteligencia emocional y el cociente intelectual con el rendimiento académico en estudiantes universitarios. Revista Electrónica de Motivación y Emoción-REME, 9(22), 1-27.

Rojas, J. V. (2014). Perfil socioeconómico e inteligencia emocional de bachilleres de nuevo ingreso del instituto universitario de tecnología de Cabimas, Venezuela. Senderos Pedagógicos (5), 13-25.

Salguero, J. M., Fernández-Berrocal, P., Aranda, D. R., y González, R. C. (2009). Propiedades psicométricas de la versión reducida de la Trait Meta-Mood Scale: TMMS-12. En Fernández-Berrocal (Ed.), Avances en el estudio de la Inteligencia Emocional (pp. 129-134). Fundación Marcelino Botín.

Schmidt, V. (2010). Las bases biológicas del Neuroticismo y la Extraversión ¿Por qué nos comportamos como lo hacemos? Psiencia Revista Latinoamericana de Evaluación Psicológica, 2(1), 20-25. doi: 10.5872/psiencia.v2i1.37
Schmidt, V., Firpo, L., Vion, D., De Costa Olivian, M. E., Casella, L., Cuenya, L., Blum, G. S., y Pedrón, V. (2010). Modelo Psicobiológico de Personalidad de Eysenck: una historia proyectada hacia el futuro. Revista Internacional de Psicología, 11(2), 1-21. doi: 10.33670/18181023.v11i02.63

Squillace, M., Janeiro, J. P., y Schmidt, V. (2013). Adaptación local del Cuestionario Revisado de Personalidad de Eysenck (Versión abreviada). Revista Evaluar, 13(1), 19-37. doi:10.35670/1667-4545. v13.n1.6794

Ugarriza, N. y Pajares-Del-Águila, L. (2001). La evaluación de la inteligencia emocional a través del inventario de BarOn (I-CE) en una muestra de Lima Metropolitana. Persona, 4, 129-160. doi:10.26439/ persona2005.n008.893

Veloso-Besio, C., Cuadra-Peralta, A., Antezana-Saguez, I., AvendañoRobledo, R., y Fuentes-Soto, L. (2013). Relación entre inteligencia emocional, satisfacción vital, felicidad subjetiva y resiliencia en funcionarios de educación especial. Estudios pedagógicos, 39(2), 355-366. doi: 10.4067/S071807052013000200022

Villanueva Acosta, V.H. (2016). Dimensiones de la personalidad $\mathrm{y}$ desordenes emocionales en estudiantes de ciencias de la salud. Acta Psicológica Peruana, 1(1) 4865. 